\title{
The influence of estrogen on female mood changes
}

Women's emotional responses can vary significantly premenstrually. They may become depressed or grumpy during menstruation or the premenstrual phase, known as premenstrual syndrome (PMS). Roughly 75\% of reproductive-age women report premenstrual mood swings or physical discomfort. Brain scans show a significant increase in activity in the medial orbitofrontal cortex related to emotional processing premenstrually, even if women's emotional responses do not vary significantly. The relationship between estrogen and emotion was first investigated over 100 years ago, and it has been shown that estrogen can improve mood. Research has shown that during prepuberty, boys are twice as likely as girls to need psychotherapy. However, the opposite is true postpubertally. Women are twice as likely to develop anxiety or depressive disorders compared to men. Women's increased vulnerability to depressive disorders typically occurs between the beginning of puberty and the age of 55, in concert with estrogen level changes. In addition, it is believed that about $95 \%$ of women have recurrent psychosis or a noticeable increase in negative emotions along with the fluctuation in endogenous estrogen level. Therefore, understanding the relationship between estrogen level changes and cyclical mood disorders can provide a theoretical basis for improving female physical and mental health.

In their paper titled "Estrogen impacts on emotion: Psychological, neuroscience and endocrine studies", Prof. Luo YueJia from the College of Brain and Cognitive Sciences of Beijing Normal University and Dr. Chen ChunPing from the Institute of Psychology of the Chinese Academy of Sciences systematically reviewed research in the fields of neuroscience, psychology and endocrinology. The paper was published in Science China Life Sciences. The authors hope the study will contribute to a better understanding of how estrogen affects female mood.

Estrogen has a wide range of effects on the body and brain. It exerts influence on the central nervous system through complex mechanisms of physiology and psychology. It can affect the generation and efficiency of neurotransmitters in the amygdala, hippocampus and prefrontal lobes, which are important brain areas related to emotion and cognition. It also plays a role in changing emotional behavior by acting on the hypothalamus-pituitary-adrenal (HPA) axis. The genetic transcription of estrogen receptors can modulate emotional behavior, and estrogen can influence emotional processing via neuropsychological factors. It enhances the coding of emotion and recognition accuracy for facial expressions. Estrogen can also affect emotional arousal and change the intensity of emotional experiences.

Clinicians have long recognized estrogen's therapeutic potential for mood change. Self-rated depression scores among oophorectomized women with depressive symptoms are significantly decreased by administration of estrogen, alone or in combination with the selective serotonin reuptake inhibitor fluvoxamine. In addition, estrogen replacement therapy is often used in postmenopausal women to improve mood, energy level and general well-being. However, estrogen is not simply a natural "physiological protectant". Some have reported that estrogen administration does not improve mood and even causes fear and anxiety. Therefore, the impact of estrogen on emotion varies and may depend on the individual's current state and the situation.

The authors believe that hormones do not exert an absolute and singular effect on the body. They regulate physical and psychological changes in numerous dimensions. Thus, the influence of estrogen on women's emotion is related to multiple systems. If we achieve a comprehensive understanding of the internal mechanisms related to emotional changes and estrogen, we can provide a theoretical support system to help address female emotional disorders. The authors also believe that the psychological, neurological and endocrine systems are interdependent. Therefore, an effective blending of psychology, biology and physiology is needed. This was the original intention of the paper.

Currently, a team led by Prof. Luo YueJia in cooperation with Prof. Huang RuiWang from South China Normal University continues to carry out related studies using electroencephalograms and magnetic resonance imaging as well as behavioral and biochemical techniques. In addition to the study of the relationship between estrogen and emotion, we have extended the research to ovarian hormones and complex social cognition.

See the article: Chen C P, Cheng D Z, Luo Y J. Estrogen impacts on emotion: Psychological, neuroscience and endocrine studies. Sci China Life Sci, 2011, 41: 1049-1062

Open Access This article is distributed under the terms of the Creative Commons Attribution License which permits any use, distribution, and reproduction in any medium, provided the original author(s) and source are credited. 